be. Throughout the period they were unable to fill all the available posts in the scientific classes, and for most of the period all the growing requirements of departments for the professional engineering and analogous classes could not be met. The biological field was the only one in which the number of good candidates was larger than the number of vacancies; but even here all the posts could not be filled since some of them required specialized ability and training. The general standard of the candidates accepted was good ; but there were not enough of really first-class quality suitable for the most exacting kinds of original work.

The system of continuous recruitment, previously tried and now adopted in all competitions for the senior scientific officer and scientific officer classes, under which candidates could apply at any time during the year, has been extended to the recruitment of professional engineers, surveyors and architects, with a consequent improvement in the position; and by further substantial salary increases and raising the upper age-limit to sixty, a sufficient number of good candidates was attracted by the beginning of 1952 to fill the reduced number of posts available, apart from those for quantity surveyors. Evidence of the diminished appeal of the Civil Service to the scientific worker or technologist is to be seen, however, in the figures given for the competitions for the scientific classes for $1949-50$ and $1950-51$. During the former year there were some 211 applications for $30-40$ posts as senior scientific officer, of whom 115 were interviewed or examined and 40 were accepted. For 1950-51 there were 262 applications for 77 posts, but only 109 were interviewed and 51 accepted. For scientific officers the trend is the same: during 1949-50, 1,196 applications for 265 posts, 745 interviewed and 285 accepted; during $1950-51,610$ applications for 398 posts, 338 interviewed and 158 accepted; and during 1951-52, 212 applications for 85 posts, 97 examined or interviewed and 59 accepted.

There is as yet little evidence on which to judge the value of the new selection methods for the administrative class. The details of the place of education and the university degree of successful candidates for the administrative class in the Home Civil Service and the senior branch of the Foreign Service during 1949-52 indicate that the new methods have made little difference to the social structure of the Service. A wide range of public and grammar schools is represented; but few schools provide more than one entrant a year. In the Foreign Service, Oxford and Cambridge are much more dominant among the universities in providing recruits than they are for the Home Civil Service. This wide distribution of schools providing candidates is possibly encouraged by the depreciation inseparable from the expansion of officialdom; but relatively lower salaries, overwork and a belief which is encouraged, for example, by Treasury parsimony on pension adjustments, that the State is a poor, if safe, employer, may well be important factors in lowering the attractiveness and prestige of the Civil Service.

The Commissioners' report includes one example of official dilatoriness which indicates the seriousness of such factors. In 1949 the Government decided to create a small new general-service class of information officers, and during $1949-50$ some 279 candidates were interviewed and 242 appointed. A special competition to fill $80-90$ posts in all grades of the class was announced in June 1951. About three thousand applications were received, and 466 candidates were interviewed. "It has not yet been possible," states the report, with unconscious irony, immediately in front of a paragraph dealing with psychologists, "to announce the results of this competition, since the future of the Information Service has come under further review."

\section{INTERNATIONAL SCIENTIFIC ABSTRACTING}

THE Abstracting Board of the International Council of Scientific Unions came into existence officially on June 1, 1952, but its activities first began about November 1951. A report on its activities to date was presented by the secretary of the Board, Prof. G.-A. Boutry, to the Executive Committee of the International Council of the Scientific Unions for its meeting at Strasbourg during July 9-10, 1953, where the Abstracting Board was itself to meet for the first time. Dispersion of the members of the Board throughout the world has caused some delay without seriously impeding the activities of the Board, apart from secretarial embarrassment, which is likely to persist until the subventions allocated to the Board permit at least one meeting in the year.

A major result achieved by the Board and noted in this report is the formal acceptance of the decisions of the United Nations Educational, Scientific and Cultural Organization and the Royal Society regarding authors' summaries by all periodicals printing papers on physics and published in the United States, the Netherlands, Belgium, France, Italy and, with a few exceptions, the United Kingdom. The Board has also requested the publishers of the more important periodicals printing papers on physics to forward a set of the final page-proofs of each of their issues to one or both of the editors of Science Abstracts and the Bulletin Analytique du Centre National de la Recherche Scientifique (Paris). This action was at first limited to a few selected periodicals in each country, and the report lists the periodicals which are co-operating in this way in the United Kingdom, the United States, France, the Netherlands, Switzerland, Canada, Australia and Japan, and further periodicals published in France, Ttaly and India, with the editors of which negotiations are still proceeding. As a net result a delay of six to eight weeks in the appearance of the most important abstracts in these two periodicals has been eliminated.

Measures have also been taken to increase the number of non-periodical publications dealt with. Physical societies have now agreed that, when an advance note of a paper or meeting is published, the account will mention the later publication of the full paper. Non-periodical publications in France will be reviewed by the editor of Bulletin Analytique, who will forward to Science Abstracts documents he considers should be abstracted, and a reciprocal service for non-periodical publications of the United Kingdom will be undertaken by the editor of Science Abstracts. Action is now being taken to extend this work to northern countries and also to cover, where necessary, colloquia and symposia organized under the auspices of the International Union of Pure and Applied Chemistry, though the Board recommends that, whenever possible, papers presented at such meetings should be published in one of the regular periodicals of physics. It is also stated in the report 
that the Physikalische Berichte, which is resuming publication, is to apply for membership of the Board and that Dr. L. H. Lampitt was attending the Strasbourg meeting as an observer on behalf of the International Union of Pure and Applied Chemistry. The lists of periodicals of physics published in the Spanish and Portuguese languages have recently been reviewed. and inquiries made with the view of compiling similar lists for Japan and the northern countries of Finland, Norway, Sweden. Denmark and the Netherlands. It has also been arranged that the Italian periodical Il Nuovo Cimento will publish in 1953 an extra number, including ten to twelve articles reviewing the work of Russian schools in certain special branches of physics.

\section{AUTOMATIC GRAIN COUNTER FOR ASSESSING QUANTITATIVELY HIGH-RESOLUTION AUTORADIO- GRAPHS}

\section{By DR. R. A. DUDLEY* and DR. S. R. PELC}

Medical Research Council Radiotherapeutic Research Unit, Himmersmith Hospital, London

A

$\mathrm{N}$ instrument for automatically counting photoA graphic silver grains has been developed to permit the use of high-resolution, low-exposure autoradiography as a quantitative method. It is expected that this extension of autoradiographic techniques to yield quantitative information will greatly increase the value of this method.

The particular autoradiograph technique for which the grain counter has been designed is that in which stripping film is employed ${ }^{1}$. In this method a spatial resolution of about $2 \mu$ can be obtained, through the use of a thin $(3-4 \mu)$, fine grain $(0 \cdot 3 \mu$ diameter) emulsion (Kodak, Ltd., NT2a), superimposed in intimate and permanent contact over the tissue section. Background is very low.

Of the various possible methods of assessing quantitatively the photographic 'image', automatic grain counting is the most suitable. Microdensitometry is not practical with this emulsion, because density is too low to permit useful measurement. For example, a grain concentration of 6 grains/100 $\mu^{2}$, which in favourable cases may be two or three times background fog, corresponds to an optical density of only 0.002 . In autoradiographs, densities as low as this would be hopelessly masked by variations in background density in other parts of the preparation, such as in the tissue section itself. Experience has shown that the use of larger grains, big enough to give a significant density, would limit the high spatial resolution which is the chief merit of the strippingfilm technique. Visual counting of grains is far too laborious as a method of quantitative measurement, except for the most specialized problems. Visual counting of tracks caused by $\beta$-particles, a possibility with the more sensitive minimum-ionization emulsions, is even more laborious and subjective than grain counting. Automatic counting of $\beta$-tracks is at present too difficult to be considered. The method of choice, automatic counting of grains, has the great advantages of high sensitivity, speed and freedom from subjective error ; its one serious disadvantage is the instrumental complexity.

* Fulbright Ssholar.
The automatic grain counter which has been built is basically similar to the flying-spot microscope and particle counter designed by Roberts ${ }^{2}$; a detailed. technical description will be published eisewhere. In brief, the operation of the instrument is as follows. A scanning spot of light is produced on a television projection tube, placed about $10 \mathrm{in}$. above the eyepiece of a microscope. This spot sweeps out an ordinary television raster (pattern of closely spaced parallel lines). The raster, projected down through the microscope (thus in the reverse direction from that used in conventional microscopy), is focused on the autoradiograph emulsion placed in the ordinary object plane of the microscope. Both scanning spot and complete raster are reduced in size by a factor equal to the reciprocal of the magnification of the microscope. With an objective having a numerical aperture of 1.3 N.A., the spot diameter can be as small as $0.3 \mu$, the size of the grains. On the autoradiograph, the full raster covers an area of $50 \mu \times$ $50 \mu$; by masking off part of the projection tube screen, it is possible to limit the scanning to any desired part of the square. Beneath the microscope condenser is a photomultiplier which continuously measures the amount of light passing through the autoradiograph. When the scanning spot crosses a silver grain, which causes 30-50 per cent absorption of the light, the brief interruption of the light beam is converted to an electrical pulse at the photomultiplier output. These pulses are then amplified, sorted according to height, and fed into a scaler. The sorting is performed by a single-channel differential-amplitude discriminator, which eliminates both those pulses which are too small (for example, noise) and those which are too large (for example, 100 per cent extinction of scanning spot during the backsweep of the spot) to be pulses caused by grains. The complete field of view is scanned once every 0.02 sec., but for greater accuracy the count is summed over about 175 successive scans in a period of 3.5 sec.

The automatic count is not absolute, although it can be calibrated by a visual count if desired. The larger grains are probably counted somewhat more frequently than the smaller ones; but this condition can be improved, if required; by careful adjustment of the discriminator levels.

Besides the counting circuits, provision is also made for a flying-spot microscope picture of the area being scanned. This is produced on a television display tube the spot of which traces out a raster synchronized with the projection-tube raster. The output from the photomultiplier controls the instant. aneous brightness of the display spot, which therefore 'paints' on its screen a picture of the field of view. A useful visual check on the automatic counting process is provided by arranging that, at the instant a count is recorded by the scaler, a brightening pulse is fed on to the display tube. The picture then shows the silver grains in the field as black dots; those grains which are being counted are followed immediately by a bright dot.

One optical problem in the counting is that the emulsion is too thick for all the grains to be in focus at once. A method of surmounting this problem is to count at several planes of focus, each separated by a small, accurately reproducible distance from its neighbours. Simple depth of focus adjustment is possible by altering the mechanical tube-length of the microscope. If a $\mathbf{9 5} \times$ objective is in use, lifting the eyepiece $1 \mathrm{~mm}$. raises the plane of focus $0 \cdot 1 \mu$. 